\title{
THE MICROSURGICAL ANATOMY OF THE GYRUS RECTUS AREA AND ITS NEUROSURGICAL IMPLICATIONS
}

\author{
Alejandro Ramos', Feres Chaddad-Neto' ${ }^{2}$ Andrei Fernandes Joaquim³, José Maria Campos-Filho', \\ João Paulo Mattos', Guilherme Carvalhal Ribas ${ }^{4}$, Evandro de Oliveira ${ }^{5}$
}

Surgical approaches to the frontal basal region as those applied to the anterior communicating artery (AntCom$\mathrm{mA}$ ) aneurysms can lead to post-operative deficits such as memory impairment, executive function disturbances and personality changes ${ }^{1-4}$. Some of such disturbances may be caused by an aneurysm rupture itself followed by vasospasm or direct injury to the brain parenquima ${ }^{3,4}$. This is especially true when we consider that many AnCommA aneurysm surgery cases are performed with a variable coagulation degree followed by the gyrus rectus aspiration (GR) in order to provide an appropriate exposure to the vessels. The knowledge concerning the pathways around the gyrus rectus is important to avoid destroying some fiber tracts with its inherent post-operative disturbances.

We reviewed the microanatomy in this area and the correlation of this zone with the limbic system, the caudate nucleus head and nucleus accumbens septi in order to establish the application of such acquired knowledge to the most common surgical procedures in this area besides formulating some important landmarks. The nervous, arterial and venous relationship of this area was also studied.

\section{METHOD}

Twenty-four adult brains were examined under magnification ranging from $6 x$ to $40 x$. The surface in all fresh brains and the white matter fiber tract dissections in the frozen specimens, the measurements among the gyrus rectus and those related structures were carried out. Twelve specimens were filled with colored latex to allow a careful study of its arterial and venous relationship with the gyrus rectus. This study was approved by the local ethics committee.

\section{RESULTS}

The results were divided into five parts: osseus, ner- vous, gyrus rectus anatomy, arterial and venous vasculature in order to provide additional information regarding the vascularization in this area; each relationship with the neighborhood structures was described.

\section{Osseous}

The orbital roof is composed of the lesser sphenoid wing and the orbital plate from the frontal bone. The sphenoid and ethmoid bones are interposed between the orbital roofs. The cribiforme plate from the ethmoid bone constitutes its medial part in its anterior $2 / 3$ and the planum esfenoidale in its posterior $1 / 3^{5}$. The surface involving this plate is longer in its anterior-posterior diameter by reaching a mean size of $20 \mathrm{~mm}$ rather than in the transversal one which is $5 \mathrm{~mm}$. The anterior skull base surface is concave in its transversal diameter more than in the anterior-posterior one with the lateral surfaces from the anterior cranial fossa being in an upper level than in its midline with its upper point almost at the tip of the crista galli process.

In the medial part, the crista galli process separates the cribiforme plate. The separation is continuous to the posterior third of the plate where the crista galli process tapers down in a wide base from a lateral point of view. The falx cerebri attaches to the crista galli process.

The lesser sphenoid wings follow a descending trajectory from the lateral to the medial part where they end as the anterior clinoid process. The concave shape at the anterior part from the anterior cranial fossa becomes flatter as it gets closer to the planum esphenoidale. The GR posterior pole corresponds with a line in its transversal plane that crosses the planum sphenoidale posterior border at the clinoid process base ${ }^{5}$.

\footnotetext{
A ANATOMIA MICROCIRÚRGICA DA REGIÃO DO GIRO RETO E SUAS IMPLICAÇÕES NEUROCIRÚRGICAS

${ }^{1} \mathrm{MD}$, Neurosurgeon Fellow of Instituto de Ciências Neurológicas (ICNE), São Paulo SP, Brazil, Neurosurgeon fellow of the Microneurosurgery Laboratory at the Hospital Beneficência Portuguesa, São Paulo SP, Brazil; ${ }^{2}$ MD, Neurosurgeon of ICNE, Neurosurgeon of the Hospital Beneficência Portuguesa, São Paulo SP, Brazil, Neurosurgeon of Universidade de Campinas (UNICAMP), Campinas SP, Brazil; ${ }^{3}$ MD, Neurosurgeon Fellow of UNICAMP; ${ }^{4}$ MD, PhD, Neurosurgeon of the Hospital Albert Einstein, São Paulo SP, Brazil; ${ }^{5} \mathrm{MD}$, PhD, Director of ICNE, Chief of the Microneurosurgery Laboratory at the Hospital Beneficência Portuguesa, São Paulo SP, Brazil, Chief of the Neurosurgery Division at UNICAMP.
}

Received 9 June 2008, received in final form 22 October 2008. Accepted 3 December 2008.

Dr. Feres Chaddad-Neto - Instituto de Ciências Neurológicas (ICNE) - Praça Amadeu Amaral 27 - 01327-010 São Paulo SP-Brasil. E-mail: fereschaddad@ hotmail.com 


\section{Nervous}

The olfactory receptor filaments give off the central fiber bundles which cross the cribiforme plate from the ethmoid bone to the olfactory bulb. The axons that leave the olfactory bulb follow a posterior direction as the olfactory tract and go along the olfactory sulcus on the orbital or basal surface at the frontal lobe. The olfactory tract bifurcates in a medial and lateral olfactory stria in front of the anterior perforated substance (Fig 1$)^{5}$.

The medial stria fibers decussate in the anterior comissure and join to its opposite mate by reaching the contralateral olfactory temporal cortex.

The lateral stria reaches the ipsilateral olfactory temporal cortex and is also connected with the amygdaloid nucleus, septal nuclei and hypothalamus.

The olfactory tract showed $31.1 \mathrm{~mm}$ in length (range 26-40 mm). The olfactory bulb and tract junction corresponded to the frontal ventricle most anterior part, the ventricular recess in a coronal plane. This landmark is located $21.3 \mathrm{~mm}$ (range 14-34 mm) anterior to the stria division and $27.3 \mathrm{~mm}$ (range 17-40 mm) posterior to the GR anterior pole.

The lateral and medial olfactory stria form a triangle or the olfactory trigone with a posterior base from the anterior limit of the anterior perforated substance (Fig 1$)^{5}$.

We found out a sulcus on the the gyrus rectus basal surface at the stria bifurcation that usually begins at the medial division initial portion of the olfactory tract and runs to the proximal part final path at the anterior cerebral artery (ACA), A1 segment, in its junction of the An-

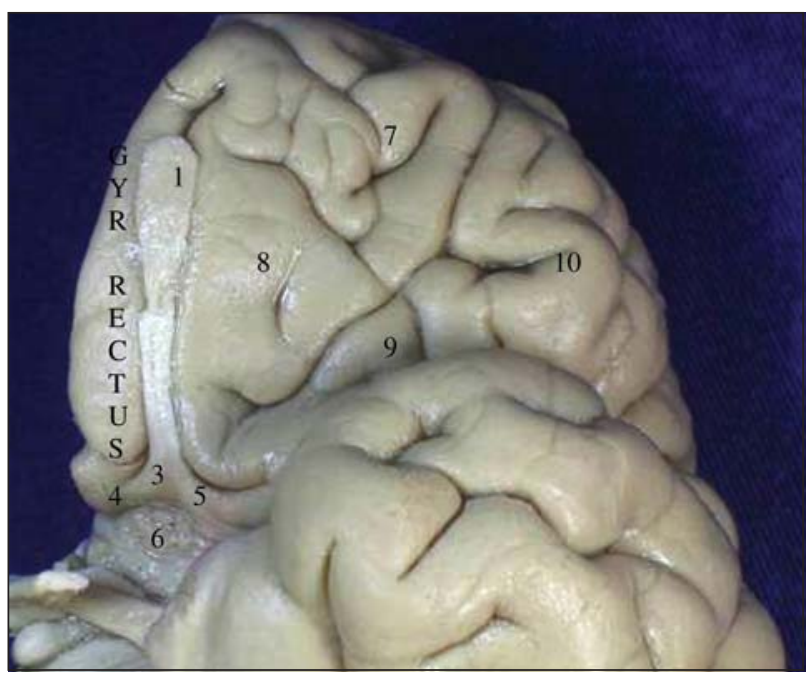

Fig 1. The olfactory nerve is divided in the olfactory bulb (1), olfactory tract (2), olfactory trigone (3) and medial (4) and a lateral (5) olfactory stria in front of the anterior perforated substance (6) in a frontal lobe basal view. The orbital surface is lateral to the olfactory tract, it is divided in the anterior orbital gyri (7), the medial orbital gyri (8), the posterior orbital gyrus (9) and lateral orbital gyri (10).

CommA (Fig 2). This sulcus is usually extended to the gyrus rectus mesial surface towards the paraolfactory area and it tends to follow the A2 segment trajectory in the ACA.

This sulcus may act as a landmark as it happened in $90 \%$ of the cases and always corresponded immediately to the A1 final trajectory before the AnCommA complex in our study. We named this finding the transverse olfactory sulcus.

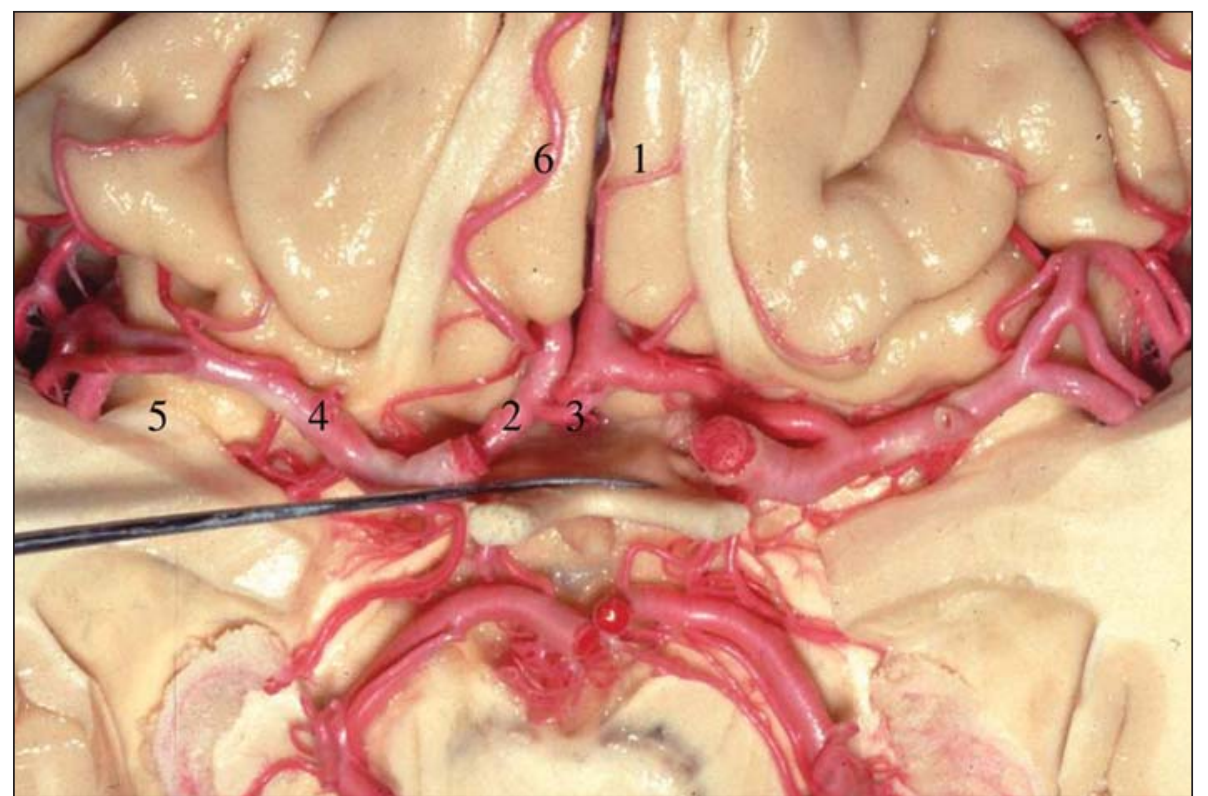

Fig 2. Basal surface of gyrus rectus (1), the anterior cerebral artery (2), the anterior communicating artery (3), the middle cerebral artery (4), the limen insula (5), the frontopolar artery (6). 


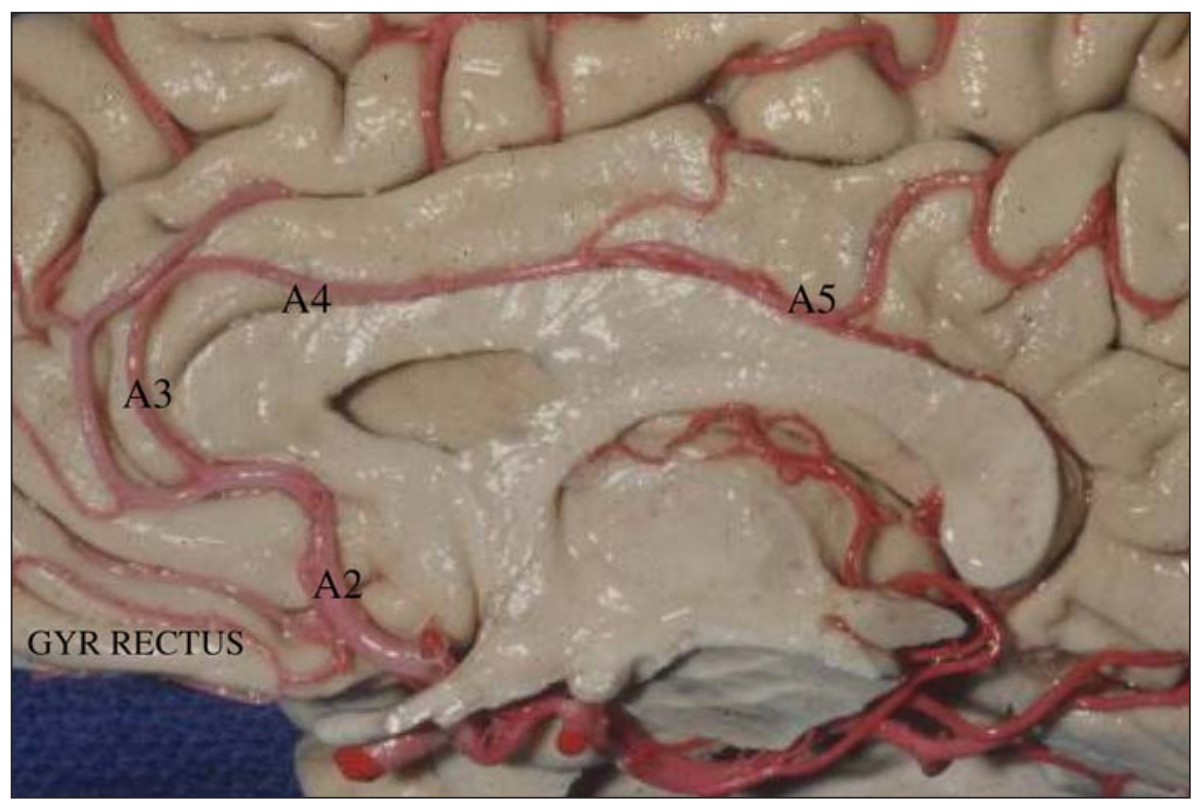

Fig 3. Medial surface of the right cerebral hemisphere, there are the gyrus rectus (GYR RECTUS) and the distal part of the anterior cerebral artery, the part beginning at the anterior communicating artery, is divided in four segments (A2, A3, A4 and A5).

\section{Gyrus rectus}

The gyrus rectus is an anatomic structure which is located at the very middle on the anterior cranial fossa floor. It also constitutes the mesial surface lowest part ${ }^{5}$ (Fig 3).

The gyrus rectus has two different association fibers: the short ones that interconnect the corpus callosum and the gyrus rectus basal and lateral surfaces. The second group is the long association fibers which interconnect the paraterminal and paraolfactory areas, the posterior third of the mesial and the adjacent basal surfaces from the gyrus rectus with the cingulate gyrus.

We can consider the GR (Brodmann area 11) as a parallelogram composed of the anterior and posterior poles, eight angles and four surfaces. Its anterior pole is previously limited to the frontal lobe convex surface by a fissure called the fronto marginal sulcus. It separates the GR quite often from the superior frontal gyrus or alternately from the middle frontal gyrus.

The GR lateral surface is limited to the olfactory sulcus where the olfactory bulb and tract overlie. The lateral surface has $9.5 \mathrm{~mm}$ in depth at the stria division (range from 6-12 mm). The olfactory sulcus separates the GR from the orbital gyri. This sulcus tended to show a medial turn in its anterior end in our study and the most frequent pattern was ending in a straight position at the posterior limit.

The inferior rostral sulcus (IRS) begins in the anterior part on the mesial surface, usually at the medial end of the fronto marginal sulcus. It was long and single in most cases. The IRS followed a posterior and lower direction with a later extension in an ascending trajectory ending in the GR posterior third usually in the cingulate pole. However, an inferior rostral sulcus accessory was usually found interrupted and very superficial below the inferior rostral sulcus in $40 \%$ of cases.

The GR mesial surface is located down to the IRS. In accordance with our dissections, the IRS depth is almost coincident with the olfactory sulcus deepest part and almost delimitating the GR as an isolated structure. The white matter fiber tract arm interposed between these sulci depth is composed of the short $U$ fibers that interconnected the GR with the orbital and the cingulate gyri. Indeed, the superior rostral sulcus (SRS) is a cingulated sulcus continuation in most cases .

The mesial surface has $5.2 \mathrm{~mm}$ in depth at the striae division (range 3-6 mm). The GR mesial surface is in contact with its anterior third at the crista galli process with the falx cerebri anterior-inferior edge. The GR tightly faces each other on the posterior mesial surface.

The gyrus rectus average length was $45.7 \mathrm{~mm}$ (range 38-58 $\mathrm{mm}$ ). The posterior pole at the basal surface has the medial stria as its posterior limit (Fig 1). The GR posterior part in its deeper and upper extension is in close relation to the anterior-inferior extension of the caudate nucleus and its infraventricular part called nucleus accumbens septi. The designation of nucleus accumbens septi was applied to all the caudate nucleus head structures located down and anterior to the anterior commisure as there is no junction between the caudate nucleus and putamen posterior and superior from the anterior comissure. The nucleus accumbens septi is located from its ini- 
tial portion immediately anterior to the anterior comissure in the paraolfactory and paraterminal gyrus depth to its $3.5 \mathrm{~mm}$ (range 2-5 mm) final part forward in the posterior third of the GR in the anterior-posterior length.

The extension of the nucleus accumbens septi lowest part into the GR is around $5 \mathrm{~mm}$ deep in the upper-lower point of view. It corresponds to the IRS projection in its mesial point of view. This nucleus accumbens septi indentation into the GR is decreased as more medial as it disappears at the most mesial part.

The ventricle recess tends to disappear in the lateral part of the ventricle in its most anterior one where it is increasingly occupied by the caudate nucleus head. The ventricle recess is separated from the GR superior limit at the olfactory bulb and tract junction with $5 \mathrm{~mm}$ in depth (range 2-8 $\mathrm{mm}$ ).

The nucleus accumbens septi mean depth in the GR posterior end laterally at the olfactory sulcus is $8 \mathrm{~mm}$ (range 6-15 mm). As you medially go further, this distance becomes as short as $5 \mathrm{~mm}$ from the basal surface, which is the lowest part of the nucleus accumbens septi indentation into the GR. In addition, this distance increases again up to the point where there is no more such nucleus indentation at the GR in its most medial extension.

The caudate nucleus head most anterior part up to the anterior comissure projects a $16.2 \mathrm{~mm}$ (range 10-22 $\mathrm{mm}$ ) point on the GR basal surface far from the stria division. The caudate nucleus head corresponds to $31 \mathrm{~mm}$ in length (range 22-38 $\mathrm{mm}$ ) on the basal surface from the gyrus rectus anterior pole.

The GR is considered the least variable gyrus in the human brain. On the other hand a bifurcation at the GR posterior third was found in two specimens (8\%) with the medial arm converging to the mesial surface with the paraolfactory gyrus continuation. There was an increased GR basal surface width around $15 \mathrm{~mm}$ at the stria division (normal width around $6.1 \mathrm{~mm}$, range $4-15 \mathrm{~mm}$ ) when this finding was observed.
The measurements performed at the gyrus rectus region at the olfactory stria division are presented in Table.

\section{Arterial relationship}

The orbitofrontal artery, which constitutes the first cortical branch in the distal ACA, is nearly all hemispheres. It commonly arises from the A2 but it may also arise as a common trunk with the frontopolar artery or be an A1 segment branch just close to the AnCommA ${ }^{6}$.

A single orbitofrontal artery coming from the $A 2$ with small branches moving from $\mathrm{A} 1$ can also be found. It goes down and forward to the gyrus rectus and usually enters the GR mesial surface from its origin.

The orbitofrontal artery is usually a single vessel at the GR posterior third and it gives off small branches which are distributed to the gyrus basal surface from mesial to lateral and from posterior to anterior. Some of the small branches cross the GR under the olfactory tract reaching as far as the orbital gyri besides supplying the gyrus rectus, the olfactory bulb, the olfactory tract and the orbital surface medial part of the frontal lobe ${ }^{6}$.

The GR also receives leptomeningeal branches from the artery of Heubner which supplies the posterior third of the gyrus. Some of such branches also supply the olfactory structures.

The nucleus accumbens septi is also supplied by the recurrent and the A1-A2 artery branches in some cases.

The subcallosal area is bordered and has its arterial supply with a variable degree of intermingled vessels coming from the orbitofrontal, frontopolar and callosomarginal arteries. If an AntCommA leptomeningeal branch named subcallosal or the corpus callosum median artery is found, it commonly supplies the paraterminal, the subcallosal gyrus, the ventrorostral part of the cingulate gyrus and the corpus callosum genu.

The mesial surface has an important relationship between two vascular structures: the orbitofrontal and the frontopolar arteries. If the A2 first bifurcation is too low,

Table. Measurements of the gyrus rectus region at the level of the olfactory stria division.

\begin{tabular}{lcc}
\hline Measures & Mean value & Range \\
\hline Lateral surface or OS depth & $9.5 \mathrm{~mm}$ & $6-12 \mathrm{~mm}$ \\
Medial surface from BS to IRS & $5.2 \mathrm{~mm}$ & $3-6 \mathrm{~mm}$ \\
BS width & $6.1 \mathrm{~mm}$ & $4-15 \mathrm{~mm}$ \\
GR length & $47.5 \mathrm{~mm}$ & $38-58 \mathrm{~mm}$ \\
Distance from the BS to NA in the lateral part of the GR & $8.3 \mathrm{~mm}$ & $5-12 \mathrm{~mm}$ \\
Distance from the BS to NA in the middle part of the GR & $5 \mathrm{~mm}$ & $5 \mathrm{~mm}$ \\
Distance of the most anterior part of the NA to the posterior pole of the GR. & $3.5 \mathrm{~mm}$ & $2-5 \mathrm{~mm}$ \\
Distance from the BS to the projection of the anterior comissure in the middle part of the GR & $8 \mathrm{~mm}$ & $5-10 \mathrm{~mm}$ \\
Distance of the projection of the VR on the BS from the stria division & $21.3 \mathrm{~mm}$ & $14-34 \mathrm{~mm}$ \\
\hline
\end{tabular}

OS, olfactory sulcus; BS, basal surface; IRS, inferior rostral sulcus; GR, gyrus rectus; NA, nucleus accumbens; VR, ventricle recess. 
the pericallosal artery follows the pericallosal sulcus. The callosomarginal artery follows the superior rostral sulcus which separates the sulcus rostralis a few millimeters lower from the A2 bifurcation in this case. The callosomarginal artery alternately crosses the cingulate gyrus to follow the cingulate sulcus when a high A2 bifurcation is found.

\section{Venous relationship}

The GR veins are divided into a superficial or anterior group draining the pole, empting the superior sagittal sinus and the posterior or deep group which courses toward the anterior perforate substance and drains into the basal vein?

The anterior orbitofrontal vein drains the gyrus rectus anterior third and the orbital gyrus adjacent parts besides emptying into the superior sagittal sinus. The olfactory vein has a trajectory paralleling the olfactory tract on the orbital surface and converging together with the paraterminal, the posterior front-orbital and the anterior cerebral veins to form the basal vein ${ }^{7}$ in the anterior perforate substance area.

The olfactory vein drains the posterior two thirds of the gyrus rectus and the medial orbital gyrus. Small veins that converge on the paraterminal vein drain the gyrus rectus mesial surface mainly in the GR posterior third.

\section{DISCUSSION}

The GR microanatomical knowledge is essential before attempting surgical dissection in this area. We found out in the GR study that there are important structures in close contact with the gyrus which cannot be underestimated.

The GR resection must be subpial and medially carried at the $A 1$ segment as it turns into the interhemispheric fissure. The final objective is to unveil the $A 2$ segment and the interhemispheric fissure contents to provide an enhanced field to deal with the AnCommA aneurysms ${ }^{8}$. This maneuver may be unsafe for the neurobehavioral outcomes after the surgery. The close relationship among the nucleus accumbens septi and the subcallosal area with the GR made this zone particularly risky.

The cognitive impairment after surgical repair of a ruptured or unruptured AnCommA aneurysms may be the result of multiple factors that involve the orbital prefrontal cortex lesions or a disconnection in the ventromedial circuits $^{1,2,9,10}$.

We have not been able to assume that patients showing the cognitive and psychiatric dysfunction post AnCommA aneurysm clipping treatment result from the extensive gyrus rectus resection, so far. Nevertheless, the close relation of the aforementioned structures to the paraolfactory area, the nucleus accumbens septi and the diagonal band of Broca made this zone extremely delicate during the AnCommA dissection procedures and other pathologies that affect this area.

The $A 1$ segment final trajectory and the $A 2$ segment initial course tended to turn into the interhemispheric fissure and follow an ascending trajectory which in most cases assumed a parallel course in front of the paraolfactory area.

The GR dissection reached the paraolfactory area as it was carried out backward to the stria division in our study. Indeed, as the transition location from the A1 to A2 and consequently the AnCommA one are usually found at the stria division, the $G R$ resection behind the $A 2$ and the stria division must be avoided.

Moreover, the nucleus accumbens septi was damaged if the dissection was performed over $5 \mathrm{~mm}$ in depth from the basal surface. Although the IRS acts as a superior limit of dissection, it is difficult to be identified for its medial location in the anterior-lateral pterional approaches. However, the GR resection of no more than $5 \mathrm{~mm}$ in its upper limit from the basal surface at the $A 1$ is a safe procedure and can be used as another landmark.

The frontal lesion resections are other important and common procedures where there are increased damage risks of important structures in close contact with the GR. The joint between the olfactory bulb and tract in the coronal plane is a safe landmark as it is always located anterior to the head caudate nucleus-nucleus accumbens septi, approximately at the anterior ventricle recess.

As far as we are concerned the wide exposure increases the surgical field and can decrease the GR surgical resection extent. It is also accomplished if an extensive cistern drainage including the lamina terminalis opening and the Sylvian fissure dissection is carried out to avoid the brain parenquima unnecessary retraction.

The pterional approach and its variations such as the orbito zigomatic can be used for clipping the AnCommA aneurysms depending on their anatomical disposition. We performed an orbito-zigomatic approach in complex AnCommA, giant and directed posterior - superior aneurysms.

In conclusion, the differentiation between the AnCommA aneurysm primary lesion after bleeding, vasospasm or isquemic related problems and the lesions caused by the surgical treatment itself are very difficult to be accomplished. However, it must be emphasized in this context that a damage to the ventral circuits can be avoided after the anatomical knowledge of this region. The neurosurgeon must search for a detailed 3D view anatomical knowledge of the brain and its relations with the neighborhood structures to offer the best possible treatment. It is judicious to have a comprehensible surgical approach 
and exposure plan which offer adequate response to the most frequent postoperative complications that may potentially cause additional morbidity for the patient when dealing with the GR pathologies.

\section{REFERENCES}

1. Botter S, Prosiegel M, Steiger H, Yassouridis A. Neurobehavioural disturbances, rehabilitation outcome, and lesion site in patients after rupture and repair of anterior communicating artery aneurysms. J Neurol Neurosurg Psychiatry 1998;65:93-102.

2. Diamond B, Deluca J, Kelley S. Memory and executive functions in amnesic and non-amnesic patients with aneurysms of the anterior communicating artery. Brain 1997;120:1015-1025.
3. Hillis A, Anderson N, Sampath P, Rigamonti D. Cognitive impairments after surgical repair of rupture and unrupture aneurysms. J Neurol Neurosurg Psychiatry 2000;69:608-615.

4. Hitter BO, Gilsbach JM. Cognitive deficits after rupture and early repair of anterior communicating artery aneurysms. Acta Neurochirur (Wien) 1992;116:6-13.

5. Rhoton Al Jr. The cerebrum. Neurosurgery 2002;51(Suppl):S1-S51.

6. Rhoton Al Jr. The supratentorial arteries. Neurosurgery 2002;51(Suppl 1):S53-S120.

7. Rhoton Al Jr. The cerebral veins. Neurosurgery 2002;51(Suppl 1):S159-S205

8. Rhoton Al Jr. Aneurysms. Neurosurgery 2002;51(Suppl 1):S121-S158.

9. Ebeling U, Cramon D. Topography of the uncinate fascicle and adjacent temporal fibers tracts. Acta Neurochirur (Wien) 1992;115:143-148.

10. Krisht AF. Anterior communicating artery aneurysms: techniques in surgical clipping. Contemp Neurosurg 2001;23:15.1-6. 